den Stellung belohnt, zum Schaden der technischen und wissenschaftlichen Angestellten.

Ich kann diese Gelegenheit nicht vorübergehen lassen, ohne etwas über die Lage der Arbeiter zu sagen. Immer, wenn über amerikanische Verhältnisse gesprochen wird, ist die ultima ratio, welche alle Unterschiede erklärt, die günstige wirtschaftliche Situation der Arbeitnehmenden. Dies trifft in hohem Grade, aber nicht ausschließlich zu. Die Lohnverhältnisse sind verschieden in verschiedenen Teilen des Landes, da Angebot und Nachfrage sich nicht sofort ausgleicht, und z. B. die den Lohn bestimmenden Gewerkschaften nicht etwa in allen Teilen gleich starken Einfluß haben. In den östlichen Industriebezirken erhält zurzeit der gewöhnliche Tagelöhner $\left.1 \frac{1}{4}-\right]^{1 / 2}$ Dollar für 10 Stunden Arbeitszeit, Handwerker $21 / 2^{1}-3^{1 / 3}$ Dollar, falls in keiner Gewerkschaft, 4 Dollar und mehr, falls in einer Gewerkschaft. Das Verhältnis von Arbeitgeber und Arbeitnehmer ist durch verschiedene Faktoren gegeben. Die demokratischen Allüren, das heilige Erbgut der amerikanischen Nation, gewähren von vornherein dem Mann in abhängiger Stellung ein Gefühl der Befriedigung, die bessere wirtschaftliche Lage gibt ihm ein Gefühl der Unabhängigkeit, die sein deutscher Bruder nicht besitzt, sie ist aber auch die Ursache, daß der Arbeitgeber glaubt, mit der Entlohnung allen seinen Verpflichtungen nachgekommen zu sein. Der Arbeiterschutz und die Wohlfahrtseinrichtungen sind minimal, und der Betrieb ist reich an Unfällen, die einer radikalen Presse zum Agitationsmaterial dienen könnten. Ein Arbeiter, der sich bei der Arbeit verletzt, hat keinen Anspruch auf Entschädigung, es sei denn, daß ein Verschulden des Arbeitgebers nachgewiesen wird, der Prozeßweg ist langwierig und teuer, und so lassen sich denn die meisten Arbeiter auf einen Vergleich ein, bei dem sie sehr zu kurz kommen. Es war und ist mir immer unerklärlich, warum die amerikanische Arbeiterschaft nicht mehr dazu tut, nach dieser Richtung Verbesserungen ins Werk zu setzen, was ihr sehr leicht fallen würde, da sie einen ausschlaggebenden Einfluß auf die gesetzgebenden Körperschaften ausübt. Es scheint fast, daß der Optimismus, den die Amerikaner mit der Muttermilch einsaugen, ihnen ein für allemal die Augen verschließt für das, was an ihren Institutionen schlecht ist. Ein patriarchalisches Verhältnis, wie man es, Gott sei Dank, hier noch so oft findet, wird man drüben vergeblich suchen.

Zum Schluß, meine Herren, möchte ich eine kleine Episode erzählen, die illustriert, mit was für Leuten der Chemiker unter Umständen zu tun hat. Ich will die Erzählung meines Gewährsmannes, der Leiter einer Schwefelsäurefabrik ist, in extenso wiederholen, um keinen wichtigen Punkt zu übergehen.

,Eines Tages“, so erzählte er mir, ,,wurde mir von meinem Chef der Auftrag zuteil, nach dem Staate Neu-York zu fahren und dort einen Kunden zu besuchen, von dem in letzter Zeit mehrere Beschwerden über die Stärke der gelieferten Schwefelsäure eingelaufen waren. Es handelte sich um eine Gerberei, und da man in kleineren Fabriken sehr häufig ganz ungenaue Aräometer findet, so nahm ich ein geeichtes Aräometer mit, um es mit dem des Kunden zu vergleichen. An meinem Bestimmungsorte an- gelangt, begab ich mich in die Gerberei und trat in die Office ein, wo sich nur einer jener echt amerikanischen „Boys" befand. Ich fragte: „Kann ich den Direktor sprechen?" ,Wen?" fragte der Junge. Ich wiederholte: ,Kann ich den Direktor sehen?" Der Junge sieht mich verständnislos an. Nach einer Weile geht ihm ein Licht auf. "Ach Sie meinen Bill", auf einen Mann in Hemdsärmeln zeigend, der sich in einiger Entfernung an einem Haufen Felle zu schaffen machte. "Gehen Sie nur darunter und rufen Sie Bill, dann wird er schon kommen." Ich tat, wie mir geheißen. Der Herr Direktor in Hemdsärmeln kam, und ich stellte mich als Vertreter der Firma vor, von der er seine Schwefelsäure bezöge. Bill führte mich zu der beanstandeten Säure, und ich fing an, mein Aräometer auszupacken. Es entspann sich nun folgendes Zwiegespräch. Ich: ,Bitte, bringen Sie Ihr Aräometer, damit wir es mit meinem vergleichen können, das geeicht ist." Bill: „Was?" Ich: ,Ich bitte um Thr Aräometer." Bill kennt offenbar den technischen Namen nicht und wiederholt: ,Was?" Ich zeige ihm mein Aräometer und sage: ,Sie haben doch so ein Glas wie dies hier, um die Stärke der Säure festzustellen. Wie können Sie sonst sagen, daß die Säure nicht stark genug ist?" Bill sieht mich geringschätzig an, rollt seinen linken Hemdsärmel in die Höhe and enthüllt einen mit Blasen und Flecken bedeckten Arm : ,, Sehen Sie, diese Blase rührt von der alten guten Säure her, aber dieser rote Fleck, das ist Thre neue Säure, die ist so schwach, daß sie keine Blasen zieht."

\section{Fortschritte in der Chemie der Gärungsgewerbe im Jahre I9o6.}

\author{
Von O. Moнr.
}

(Eingeg. d. 14.|3. 1906.)

\section{Chemieder Rohstoffe.}

Auf das Jahr 1905, dessen G e r s te n qualitativ nicht sehr befriedigend waren, ist mit dem Berichtsjahr ${ }^{1}$ ) wieder ein besseres Jahr gefolgt. Vor allen Dingen ist der Eiweißgehalt der Gersten erheblich niedriger wie im Vorjahr; ist auch die Farbe der Gersten meist nicht gerade hervorragend, so fehlen doch Braun- und Dunkelspitzigkeit fast völlig. Die Keimfähigkeit ist eine hervorragend gute. Nach alledem kann man mit ziemlicher Sicherheit annehmen, daß die Weiterverarbeitung zu Malz und weiter zn Bier keine unvorhergesehenen Schwierigkeiten machen wird, wie es namentlich bei den abnorm eiweißarmen Gersten des Jahres 1904 der Fall war. Zu dem so vielfach erörterten Thema des Eiwei $B$ es in der Gers te liefert E. Prior ${ }^{2}$ ) eine umfangreiche Arbeit über die Bedeutung der Gerstenproteïde für die Bewertung und ihre Beziehungen zur Glasigkeit der Gerste. Verf. hat nach den seinerzeit von $\mathrm{Os}$ b o r ne angegebenen $\mathrm{Me}$ thoden die Hauptgruppen der Proteïde getrennt bestimmt und zieht aus den Ergebnissen seiner Arbeit unter anderen folgende Schlüsse: Der Ge-

1) Wochenschr. f. Brauerei 23, 611 (1906).

2) Allg. Z. f. Bierbrauerei 1906, Novemberheft. 
halt der Gerste an wasserlöslichen Proteiden und an Edestin steht in keiner festen Beziehung zum Gesamtstickstoffgehalt, dagegen steigt und fällt der Gehalt an Hordeïn und unlöslichem Proteïd mit steigendem und fallendem Gesamteiweiß. Für die Bewertung der Gerste ist der Gehalt an Hordeïn und unlöslichem Proteïd entscheidend. (Demgemäß ist die umständliche Trennung der Eiweißstoffe bei der Gerstenanalyse überflüssig, und es genügt, wie bisher üblich, die einfache Bestimmung des Gesamtstickstoffs. Der Referent.) Die Scheinglasigkeit wird durch Verkitten der stärkeführenden Zellen mittels stickstofffreien und stickstoffhaltigen Kolloidstoffen verursacht, die echte, schädliche Glasigkeit besteht in einer Verkittung der Zellen durch Hordeïn und unlösliches Proteid. Die Umwandlung der scheinglasigen Körner in mehlige wird stark von der Zusammensetzung des Weichwassers beeinflußt, günstig wirkt hierbei Gipsgehalt. $\mathrm{stockmeier}$ und $W$ olfs ${ }^{3}$ ) haben in einer größeren Zahl Gersten Stiokst of f - und Extraktgehalt bestimmt. Thr Befund, daß fast in allen Fällen steigender Stickstoffgehalt mit fallendem Extraktgehalt Hand in Hand geht, ist eine weitere Stütze für die bekannte $\mathrm{H}$ a a s e sche Anschauung über die Beziehungen zwischen Stickstoffgehalt der Gerste und Extraktgehalt des daraus gewonnenen Malzes. Unter diesen Umständen ist eine Methode von E. J a low et $z^{4}$ ) zur raschen Orientierung über den $S t$ i ckst of f g e halt in Gerstenkörnern von Interesse. Die Körner werden bei der Temperatur des siedenden Wasserbades ca. 30 Minuten in Formalin erhitzt und nach mehrfachem Abspülen mit kaltem Wasser der Schnittprobe unterzogen. Alle Körner zeigen einen dünnen, gummiartigen Rand, der entweder eine mehlige, eine halbglasige oder eine glasige Mitte. umgibt. Stickstoffarme Körner sind mehlig, mit dem Stickstoffgehalt wächst die Glasigkeit.

Uber eine Methode zur Ex tra ktbestim $\mathrm{mung}$ in Gerste berichtet $\mathrm{H}$. Graf ${ }^{5}$ ). In den Gerstenspelzen hat $H$ Seyfert ${ }^{6}$ ) außer bereits früher beschriebenem wasserunlös. lichem Gerbstoff noch einen wasserlöslichen, ziemlich leicht veränderlichen gefunden, dessen Lösung alkalische Kupferoxydlösung reduziert. Als weitere Bestandteile kommen Bitterharze sauren Charakters vor, die sich in reinem Wasser schwer, in carbonathaltigem Wasser vermöge ihres Säurecharakters leicht lösen. Bemerkenswert ist ihre Giftigkeit für Schimmelpilze und Bakterien.

Ein Getreideprüfer nach Frank$\mathrm{Kamenetzky}{ }^{7}$ ) läßt an der Skala direkt das Hektolitergewicht ablesen.

Die so sehr verschiedene Haltbarkeit der Kartoffeln beim Lagern hat W. He n n e be r g ${ }^{8}$ ) zu Versuchen über die Widerstandsfähigkeit verschiedener Kartoffelsorten ge gen über Fäulnisbakterien veranlaßt. Wenn auch eine völlige Aufklärung der Frage nicht

3) Z. ges. Brauwesen 29, 252 (1906)

4) Allg. Z. f. Bierbrauerei 1906, 41.

5) Z. ges. Brauwesen 29, 25 (1906).

6) Wochenschr. f. Brauerei $\mathbf{2 3}, 545$ (1906).

7) 7. ges. Brauwesen 29, 114 (1906).

8) Z. f. Spiritusind. 29, 52 (1906). gelang, bei der jedenfalls eine ganze Reihe Faktoren eine Rolle spielen, so zeigte sich doch mit voller Sicherheit daß der Zuckergehalt der Kartoffeln von wesentlichem Einfluß ist; je höher dieser Gehalt, desto geringer im allgemeinen die Haltbarkeit.

v. d. Heide ${ }^{9}$ ) hat nach dem Prinzip der $\mathrm{N}$ i c hols on schen Senkwage einen Apparat zur Bestimmung des Stärkegehaltes in de $\mathrm{n}$ K a $\mathbf{r}$ of $\mathrm{f}$ e $1 \mathrm{n}$ konstruiert. Die Eintauchtiefe läßt am Stengel des Instrumentes direkt die Stärkeprozente ablesen.

Finen neuen brauchbaren $\mathrm{R}$ ohs tof f $\mathrm{zu} r$ Alkoholbereitung stellt nach E. A. M a $n n^{10}$ ) das Mark eines westaustralischen Baumes, Xanthorrhoea Preisii, dar, das 20\% vergärbaren Zucker enthält.

\section{Mälzerei, Malz, Malzunter- suchung.}

Um auch stickstoffreiche Gerste zu brauchbarem Malz zu verarbeiten, ist es nach W. Wi n dis e h ${ }^{11}$ ) nötig, beim Mälzen einen $\mathrm{zu}$ weit gehenden Eiweißabbau zu vermeiden. Man erreicht den gewünschten Zweck durch Arbeiten auf kurzes Gewächs. H. S e y f fe r t 12) spricht dem Weichwas s e r entscheidenden Einfluß für das Zustandekommen eines bestimmten $\mathrm{M} \mathrm{a} \mathrm{l} \mathrm{z}$ charakters.zu. Er will typische Malze, wie Pilsener, Münchener usw. Malz dadurch erzielen, daß er durch Zugabe von Calcium- resp. Magnesiumcarbonat die Weichwässer ähnlich zusammengesetzt gestaltet, wie es die Pilsener, Münchener usw. Brauwässer sind. Durch Verbindung der $\mathrm{M} \mathrm{a} \mathrm{l} \mathrm{z} \mathrm{d} \mathrm{a} \mathrm{r} \mathrm{re}$ m it ein er $W$ a g e hat $K$. Gut $t$ w e in ${ }^{13}$ ) eine Vorrichtung geschaffen, welche eine scharfe $\mathrm{R} \mathrm{e} \mathrm{g} \mathrm{u} \mathrm{-}$ lierungdes Wassergehaltes und damit des Farbegrades und bis zu einem gewissen Grade des Charakters des Malzes beim. Darren gestattet.

W. Wind is eh und W. Vogels a n g 14) haben über die Art der Phosphorverbin. dungen in der Gerste und deren Verän derungen während des Weich-, Mälz-, Darr-und Mais chprozesses gearbeitet. Danach sind in der Gerste anorganische Phosphorsäureverbindungen überhaupt nicht enthalten, solche bilden sich erst bei Wassergegenwart durch enzymatische Hydrolyse dieser organischen Phosphorverbindungen. Die Keimung der Gerste ist mit dem Abbau der organischen Verbindungen verbunden, der ganz besonders stark bei der Vermaischung des Malzes verläuft. Diese Befunde stehen im Einklang mit Angaben von $\mathrm{Z}$ a $\mathrm{l}$ e s $\mathrm{k}$ i 15), der ebenfalls bei der Keimung von Samen eine enzymatische Umwandlung organischer Phosphorverbindungen in anorganische Phosphate beobachtet hat.

Ausführliche Untersuchungen über $\mathrm{MaIzdiastase}$ veröffentlicht A. Kle e.

9) Z. f. Spiritusind. 29. 473 (1906).

10) J. Soc. Chem. Ind. 1906, 1076, nach Z. f. Spiritusind. 30, 3 (1907).

11) Jahrb. Vers. u. Lehranst. f. Branerei 9, 30 (1906).

12) D. R. P. 173475

13) Wochenschr. f. Brauerei 23, 296 (1906).

14) Wochenschr. f. Brauerei 23, 516, 556 (1906)

15) Ber. Deutsch. Botan. Gesellsch. 1906, 285. 
$\mathrm{m}$ a $\left.\mathrm{n}^{16}\right)$. Er bestimmt nach abgeänderten Methoden das Stärkeumwandlungs- und das Zuckerbildungsvermögen. Das erstere - kurz F.J. bezeichnet - wird ausgedrückt durch die Anzahl Teile wasserfreier löslicher Stärke, welche durch einen Teil Malztrockensubstanz in jodindifferente Körper verwandelt werden; das letztere - kurz F. M. bezeichnet - durch die Anzahl Teile derselben Stärke, welche durch einen Teil Malztrockensubstanz zu $77,5 \%$ in Maltose verwandelt werden. Bei gutem Grünmalz ist F. J. 3-3,2mal größer als F. M., bei vier untersuchten Darrmalzen war F. J. 3,28-5mal größer als F. M. Mit Hilfe dieser Methoden hat Verf. zu ermitteln versucht, in welcher Weise die Diastasebildung vom Wassergehalt der Gerste abhängig ist. Nach seinen Versuchen soll es für eine und dieselbe Gerste für jede Keimtemperatur einen bestimmten Wassergehalt geben, bei dem die stärkste Diastasebildung stattfindet. G. E1l rodt ${ }^{17}$ ) kann die von M. D e l b r ü $\mathrm{ck}$ schon vor Jahren ausgesprochene Vermutung experimentell bestätigen, daß klein körnige Gersten M a l z mit gröB e rem Diastasegehalt liefern als großkörnige. Der Grund für diese Erscheinung ist darin $\mathrm{zu}$ suchen, daß bei kleinkörnigen Gersten auf die Gewichtscinheit eine größere Anzahl Keimlinge kommt, und die Zah] der Keimlinge entscheidend ist für die Intensität der enzymatischen Wirkungen im Korn. Nach L. M a qu en n e und E. $\mathbf{R}$ o $\mathrm{x}^{18}$ ) steigert sich die $\mathrm{W}$ i r k a $\mathrm{mk}$ e it $\mathrm{von}$ kalt bereiteten Malzauszügen beim Stehen sehr bedeutend, wahrscheinlich infolge einer Art Proteolyse von Zymogenen. Notwendig ist dabei ein Sterilhalten des Auszuges durch Zugabe von Antiseptica.

Im Gegensatz zur keimenden Gerste soll D a r r$\mathrm{malz}$ nach $\mathrm{H}$. und $\mathrm{A}$. $\mathrm{E} \mathrm{u} \mathrm{I}$ e ${ }^{19}$ ) keine $\mathrm{Oxy}-$ ge n a se, sondern nur Katalase und Peroxydase enthalten. Wie B a ch gezeigt hat, sind die Oxygenasen sehr leicht zerstörbare Körper, möglicherweise werden sie beim Darren des Malzes vernichtet. Wie L. M a rino und G. Fi.o rentin o ${ }^{20}$ ) behaupten, coll die Maltase a us MalzauBer Maltose auch die durch Emulsin spaltbaren natürlichen und künstlichen Glucoside spalten. Nach der von den Verff. benutzten Darstellungsmethode der Maltase liegt aber in dem Präparat zweifellos ein Gemisch aller möglichen Malzenzyme vor, so daß die beobachteten Enzymwirkungen richtiger auf dieses Enzymgemisch zurückzuführen sind.

Aus $\mathrm{Malzk}$ ei m e $\mathbf{n}$ hat E. Lég e $\mathbf{r}^{21}$ ) nach dem $\mathrm{S}$ t a s schen Verfahren ein Alkaloid, das $\mathrm{H}$ or denin, von der Gesamtformel $\mathrm{C}_{10} \mathrm{H}_{15} \mathrm{ON}$ isoliert. Der Körper stellt eine starke tertiäre Base dar, die Ammoniumsalze zersetzt und wohlcharakterisierte Salze, Acetylverbindungen usw. liefert. Das Sulfat wirkt nach $\mathrm{L}$. $\mathrm{Ca} \mathrm{m} \mathrm{u} \mathrm{s}{ }^{22}$ ) schwach giftig. Das ge-

16) Landw. Vers.-Stat. 63, 93 (1906).

17) Wochenschr. f. Brauerei 23, 243 (1906). (1906).

18 ) Compt. r. d. Acad. d. sciences 142, 1387

19) Arkiv f. Kemi 1, 365 (1906); vgl. Chem. Centralbl. 1906, I, 1107.

20) Gaz. chim. ital. 36, II, 395 (1906).

21) Compt. r. d. Acad. d. sciences 142, 108; 143, 234, 916 (1906).

22) Compt. r. d. Acad. d. sciences 142, 110. nauere Studium hat das AJkaloid als p-Oxyphenyläthyldimethylamin $\mathrm{HO} \cdot \mathrm{C}_{6} \mathrm{H}_{4} \cdot \mathrm{C}_{2} \mathrm{H}_{4} \cdot \mathrm{N}\left(\mathrm{CH}_{3}\right)_{2}$ erkennen iassen. Den gleichen Befund hat unabhängig von Léger auch $O$. Ga e bel ${ }^{23}$ ) gemacht.

Malze für du $\mathrm{n}$ le $\mathrm{B}$ i e re zeigen nach C. L i $n t n$ e $\mathbf{r}^{24}$ ) bisweilen im Laboratorium abnorm lange Verzuckerungszeiten, ohne jedoch bei der Verarbeitung in der Praxis Schwierigkeiten zu machen. Die erzielten Würzen haben hohen Nichtzuckergehalt, es verdienen daher solche Malze Beachtung zur Herstellung niedrig vergorener Biere. Die Minderextraktausbeute aus dunk l e $n \mathrm{M}$ a $\mathrm{l}$ z e $\mathbf{n}$ gegenüber hellen Malzen aus derselben Gerste wird nach $W . W$ in d is $\mathrm{ch}{ }^{25}$ ) z. T'. durch größere Substanzverluste durch Atmung und Röstung auf der Darre bedingt, z. T. ist aber die Minderausbeute nur eine scheinbare, indem die dextrinrejcheren dunklen Würzen im Vergleich zu gleichkonzentrierten zuckerreichen hollen Würzen ein geringeres spez. Gew, zeigen. $\mathrm{O} . \mathrm{M} \circ \mathrm{h} \mathrm{r}{ }^{26}$ ) gibt Tabellen zur Extraktbestim mung bi Malzanalysen mittels des ZeiBschen Ein. ta u chefraktometers. Weitere Tabellen desselben Verf. 27) ermöglichen die Anwendung dieses Apparates auch bei der Untersuchung von Bctriebswürzen.

\section{Sudhausarbeit, $\mathrm{M}$ a is ch proze $\beta$}

Wie Pankrath ${ }^{28}$ ) zeigt, ist die $\mathrm{Zusam}$ mensetzungder Brau ässer von merkbarem EinfluB auf die Extraktausbeute i $\mathrm{m} \mathrm{Sudh}$ a u s. Bei vergleichenden Versuchen mit gipsreichem Brunnenwasser und calciumearbonatreichem Quellwasser ergab das erstere aus Grobschrot die besten Ausbeuten, mit letzterem blieb die Ausbeute erheblich hinter der Laboratoriumsausbeute zurück. Auch die Vergärung war eine verschiedene, bei den Gipswürzen war der Endvergä. rungsgrad niedriger wie bei den anderen. Eine in der Hauptsache englische Verhältnisse berührende Arbeit über die Unterschiede der Dampf- und Feuerkochung stammt von J. Can n on und W. F y ffe ${ }^{29}$ ). Von allgemein interessierenden Ergebnissen der Arbeit ist unter anderen die Beobachtung zu erwähnen, daß unter sonst gleichen Bedingungen die Eiweißstoffe in Dampf- und Feuerwürzen sowie in den daraus gewonnenen Bieren sich insofern unterscheiden, als die Dampfwürzen und -biere reicher an echten Eiweißstoffen sind. Amide und Peptone sind in beiden Würzen annähernd in gleicher Menge enthalten; nach der Gärung sind die Dampfbiero reicher an Peptonen und ärmer an Amiden wie die Feuerbicre. Diese letztere Angabe bedarf noch der Bestätigung, ein erhöhter Amidgehalt müßte nach den bisherigen Erfahrungen eine geringere Haltbarkeit der Feuerbiere bedingen, während die Verff. gerade das Gegenteil beobachten.

Ein rasches Abläutern and ein voll

23) Arch. d. Pharmacie 244, 435 (1906).

24) Z. ges. Brauwesen 29, 637 (1906). (1906).

$\left.{ }^{25}\right)$ Jahrb. Vers. u. Lehranst. f. Branerei 9, 36

26) Wochenschr. f. Brauerei 23, 136 (1906).

27) Wochenschr. f. Brauerei 23, 609 (1906).

28) Z. ges. Brauwesen 29, 680 (1906).

$\left.{ }^{29}\right)$ J. of the Instit. of Brew. 1906, 39; durch Wochenschr. f. Brauerei 23, 326 (1906). 
ständiges Extrahieren der Treber soll das Abläuterverfahren nach $\mathrm{Hellw}$ ig - W e y g a $n d t^{30}$ ) gewährleisten. Das Verfahren besteht darin, daß während des ganzen Abläuterns der Nachgüsse die Treber mit immer tiefer eingreifenden Messern horizontal durchschnitten werden. Das Cberschwänzen vollzieht sich kontinuierlich.

Ganze Getreidekörner, z. B. Mais, kann man ohne Vorbehandlung $\mathrm{d}$ ä $\mathrm{m}$ p e $\mathrm{n}$; man bringt dieselben nach $H . A . H$ ü $b n$ e ${ }^{31}$ ) rasch in den mit kochendem Wasser beschickten Dämpfer, schließt diesen und steigert durch einströmenden Dampf den Druck zunächst auf $2-3$, zum Schluß auf 4 Atmosphären.

Nach A. Boid in ${ }^{32}$ ) kann man die früher beim Amyloverfahren zur VerfIüssi. $\mathrm{g} \mathrm{u} \mathrm{n}$ g d e r $\mathrm{M}$ a i s $\mathrm{c}$ h e verwendeten Mengen Malz vollkommen sparen, wenn man das zu vermaischende Getreide mit Säure einige Zeit einweicht und dann dämpft. Die Säuregabe ist so $\mathrm{zu}$ bemesssen, das die im Getreide vorhandenen mehrbasischen Phosphate (? siehe die oben erwähnten Arbeiten von Windisch, Vogelsang und Zaleskil Der Referent.) in einbasische verwandelt werden.

\section{Gärungsorganismen und Gär ungsvorgang.}

Auch im Berichtsjahr ist die Anzahl der veröffentlichten Arbeiten, welche dieser Uberschrift unterzuordnen sind, eine so ungeheuer zahlreiche, daß an dieser Stelle nur über eine Auswahl dieser Arbeiten berichtet werden kann.

Allgemeine Gesichtspunkte entwickelt M. D e I $\mathrm{b} r$ ü $\mathrm{e} \mathrm{k}^{33}$ ) in einem Vortrag über den $\mathrm{ph}$ y s i o logisohen Zustand der Zelle und seine Bedeutung für die Technolo. gi e der Gärungsgewerbe. Das Leben der Einzelzelle wie des Gesamtorganismus ist an eine geregelte Enzymtätigkeit gebunden, aufbauende und abbauende Wirkungen müssen sich das Gleichgewicht halten. Beim Utbergang in Ruhezustände, wie beim Reifen der Früchte überwiegen die aufbauenden Enzymtätịkeiten. Zu reichlicher Enzymgehalt bewirkt bei den Organismen den Zustand der „Hitzigkeit" - hitzige Gerste, hitzige Kartoffeln, hitzige Hefe. Hitzige Stoffe unterliegen der Gefahr der Selbstauflösung und sind Infektionen leicht zugängig. Die Enzymabsonderung kann durch Reizstoffe und durch Reizwirkungen (erhöhte Tempera. tur, Lüftung) beeinflußt werden. Die Stoffe, welche durch die Enzymtätigkeit gebildet werden, können unter Umständen dem Organismus als Kampfmittel gegen andere Organismen dienen (Kampfenzyme). Die experimentellen Arbeiten auf dem besprochenen Gebiet haben Verf. und seine Mitarbeiter L a $\mathbf{n} \mathrm{g}$ e, Henneberg, Hayduck und Wende I ${ }^{34}$ ) eigenartige $G$ if t $w$ ir $k$ ungen gewisser $G$ e treidesorten a $\mathrm{f} \mathrm{H}$ efen kennen gelehrt. Der Giftstoff gehört augenscheinlich zu den Eiweißstoffen, auch isolierte Eiweißstoffe, wie Hühner(1906).

30) Jahrb. Vers. u. Lehranst. f. Brauerei 9, 35

31) D. R. P. 166628

32) Rev. chim. pure et appl. 9, 194 (1906).

33) Wochenschr. f. Brauerei 33, 513 (1906) (1906).

34) Jahrb. Vers. u. Lehranst. f. Brauerei 9, 27

Ch. $190 \pi$. eiweiß, Weizenkleber, Handelspeptone üben solche Giftwirkungen aus. Anorganische Salze, vor allem Calcium- und Magnesiumsalze heben die Giftwirkung auf. Verschiedene Heferassen sind verschieden empfindlich, bei ein und derselben Rasse ist der physiologische Zustand von erheblichem Einfluß auf den Grad der Empfindlichkeit.

Die viel erörterte $B$ i os fra g e ist auch im Berichtsjahr Gegenstand mehrfacher Bearbeitung gewesen, und zwar mit demselben Erfolg wie bislang: in ihren Schlußfolgerungen widersprechen sich die verschiedenen Bearbeiter. H. Pr in g s h e i m ${ }^{35}$ ) leugnet die Existenz des geh€imnisvollen Stoffes, er erklärt das Nichtangehen sehr geringer Hefeimpfungen in lediglich mincralsalzhaltigen Zuckerlösungen mit der Annahme, daß Hefe anorganisch gebundenen Stickstoff, Schwefel und Phosphor erst nach erfolgter Gewöhnung assimilieren könne. Bei großen Impfgaben können die z. T, absterbenden Zellen die übrig bleibenden in der Gewöhnungszeit mit organischem Stickstoff- usw. Verbindungen versorgen, denselben Erfolg hat bei sehr geringer Hefeimpfung die Zugabe von etwas Hefeabkochung. Demgegenüber hält $\mathrm{A}$. $\mathrm{K}$ ossow i e z ${ }^{36}$ ) an der Wild i e rs schen Bioshypothese fest. Nach seinen Beobachtungen können sehr geringe Mengen Schimmelpilze, Mycoderma usw. die Entwicklung kleinster Hefeimpfungen in rein mineralsalzhaltigen Zuckerlösungen ermöglichen; Verf. bezeichnet diese Organismen direkt als Biosbildner. Das Gelingen der $\mathrm{P}$ a s t e u r schen Versuche, in Nährsalzlösungen mit Ammoniumsalzen als einziger Stickstoffnahrung kräftige Gärung zu erzielen, will Verf. auf die Gegenwart derartiger Mikroorganismen zurückführen. Die Einwirkung von Reizstoffen auf die Eigenschaften und die Tätigkeit der Hefe behandelt eine Arbeit von F. Schoen feld ${ }^{37}$ ). Die Behandlung der Hefe mit phosphathaltigem Wasser ändert die Art und Weise des Absetzens der Hefe: nach der Phosphatbehandlung bildet sie keinen festen Bodensatz mehr nach beendeter Gärung; eine Beschleunigung der Gärung wurde nicht beobachtet. Zusatz von sterilisiertem Malzmehl zur Hefe erhöhte den Vergärungsgrad außerordentlich, auch nach mehrmaliger Führung unterschied sich die so behandelte Hefe noch durch höheren Vergärungsgrad von der unbehandelten Hefe.

In früheren Arbeiten haben P. L i n d n e $\mathbf{r}^{\mathbf{3}}$ ) und seine Mitarbeiter gezeigt, $\mathrm{daB}$ die Selb st verdau unsprodukte der $H$ efe von verschiedenen Hefen verschieden as s imiliert werden. Mit dem größten Assimilationsvermögen begabt waren die luftliebenden, wenig oder keine Gärung erregenden Hefen, dann folgten Nachgärungshefen, schlieBlich die Kulturbierhefe. Bei Untersuchung einer größeren Anzahl weiterer Mikroorganismen sehen P. L in d n e r und F. S t o c k h a u s e ${ }^{39}$ ) diesen Befund bestätigt. B e ta in, das sich in den Melassen in merkbaren Mengen findet, ist nach $G$. H e inze $1 \mathrm{~m}$ a $n n^{40}$ ) für Hefe nicht

35) Z. Bakt. u. Paras. 16, II, 111 (1906).

36) Z. Österr. landw. Vers.-Wes. 9, 688 (1906) $(1906)$

37) Jahrb. Vers. u. Lehranst. f. Brauerei 9, 46

38) Diese Z. 19, 620 (1906).

39) Wochenschr. f. Brauerei 83, 519 (1906).

40) Jahrb. Ver. Spiritusfabr. 6, 22 (1906). 
a s s i m i l i e r b a r, das Chlorhydrat schädigt sogar die Hefe, wahrscheinlich infolge hydrolytischer Spaltung des Salzes in Betain und Chlorwasserstoff.

Die Anpassung der Hefe an schwef$\mathrm{l}$ i g e $\mathrm{S}$ ä u re soll nach G. Gi m e $\mathbf{l}^{\mathbf{4 1}}$ ) in einer $\mathrm{Zu}$ nahme der oxydativen Kraft der Zelle bestehen. Z.T. scheint die schweflige Säure in Form von Schwefelsäure durch die Zelle gebunden zu werden, jedenfalls wurde in der Asche von akklimatisierter Hefe mehr Sulfat gefunden, wie in der Asche von nicht akklimatisierter Hefe. E. P o z z i - E s o o t ${ }^{42}$ ) hält demgegenüber seine alte Anschauung aufrecht, daß sich die Hefe durch hydrogenisierende Wirkung gegen die Giftwirkung der schwefligen Säure schützt.

In Fortsetzung seiner Arbeiten ${ }^{43}$ ) über die Brauchbarkeit der Ameisensäure als Desin fekt ionsm it tel hat W. Henneber g $\mathrm{g}^{44}$ ) die Giftwirkung dieser Säure auf verschiedene Pilze studiert, mit denen er Maischen infiziert hat. Auch bei diesen 'Versuchen zeigte sich die Kulturhefe außerordentlich viel widerstandsfähiger gegen die Säure wie die übrigen Mikroorganismen, allem Anschein nach läßt sich ihre Widerstandsfähigkeit durch Anpassung noch weiter steigern. Derselbe Verf.45) hat noch eine Reihe weiterer Stoffe, Mineralund organische Säuren, Alkohol, Natronlauge, Formaldehyd bezüglich ihres Einflusses auf infizierte Brennerei- und Preßhefe untersucht. Auf Grund der dabei ermittelten verschiedenen Widerstandsfähigkeit der für Hefeinfektion in Frage kommenden Mikroorganismen macht Verf. Vorschläge zur Benutzung der genannten Stoffe zum Waschen der Hefe und zur Durchfïhrung von Reinigungsgärungen.

Th. B ok orn $\mathrm{y}^{46}$ ) hat die $\mathrm{G}$ if $\mathrm{tm}$ en $\mathrm{g}$ e $\mathrm{n}$ $z u$ ermitteln versucht, welche zur Abtötung einer bestimmten Menge lebender Substanz nötig sind. Die Versuche wurden meist mit Preßhefe angestellt. Zunächst wurde die eben noch wirksame Giftkonzentration ermittelt und dann die absolute Giftmenge, die zur Tötung nötig ist. Wesentlich neue Ergebnisse hat die Arbeit nicht gebracht.

In ausführlicher Arbeit berichten F. S e h o e n feld und W. Rom mel ${ }^{47}$ ) über dic Unter schiede zweier Bierhefenrassen D u n $\mathrm{d} \mathrm{K}$ in morphologischer, physiologischer, physikalischer und chemischer Hinsicht. Der Eiweißgehalt ist bei der höher vergärenden Hefe $K$ höher wie bei der Hefe D, dasselbe gilt vom Aschen- und Phosphorsäuregehalt. Hefe D zeigt größere Neigung zur Glykogenbildung. Gegen höhere Temperatur ist Hefe $\mathrm{K}$ empfindlicher, die relativ größte Temperaturwiderstandsfähigkeit zeigen beide Hefen $2-4$ Tage nach dem Anstellen mit Würze. Zum N a c h weis von Sarcinen benutzen Bethges

41) Bll. de l'Assoc. d. Chim. de Sucr. et Dist. 23, 669 (1906).

42) Bli, de l'Assoc. d. Chim. de Sucr. et Dist. 23, $1021(1906)$.

43) Diese Z. 19, 622 (1906).

44) Z. f. Spiritusind. 29, 34 (1906)

45) Wochenschr. f. Brauerei 23, 527 (1906).

46) Pharm. Centralh. 4\%, 12Iff. (1906).

47) Wochenschr. f. Brauerei 23, 523ff. (1906). und $\mathrm{H}$ e $\mathrm{ll}$ e $\mathrm{r}^{48}$ ) ungehopftes, endvergorenes, kleistertrübes Bier.

P. L i n d $n$ e ${ }^{49}$ ) hat neuere Untersuchungen über den We i n buketts chi m mel (Sachsia suaveolens) angestellt. AuBerordentlich weit ver gärend, übertrifft er im Vergärungsvermögen selbst Schizosaccharomyces Pombe, wird aber darin von der Logoshefe übertroffen. Er vergärt außer den Hexosen Saccharose, Maltose, Lactose, Raffinose, Dextrin, $\beta$-Methylglucosid, unvergoren bleiben $\alpha$-Methylglucosid, Inulin, Trehalose, Melibiose (? Der Ref.), die Pentosen, Heptosen usw. Über die Verwendung von Sachsia zur Herstellung alkohol freier Getränke ist seinerzeit50) berichtet worden. Nach P. Vi a l a und P. P a cot te t ${ }^{51}$ ) kann aus den Mycelfäden von Gloeosporium nervisequum eine Sproßhefe mit schwachem Alkoholbildungsvermögen hervorgehen.

In einer Arbeit: Zur Kenntnis der Schnellessig- und Weinessigbakterien beschreibt W. Hen $n$ eberg ${ }^{52}$ ) eine Anzahl neuer Essigbakterien und macht weiter Angaben über ihr Verhalten gegenüber verschiedenen Essigsäure- und Alkoholmengen, über ihr Säuerungs. vermögen usw. Auffallenderweise sind die Bakterien sehr empfindlich gegen Essigsäure, die von Anfang an vorhanden ist, gegen die durch die Essiggärung später entstehende Säure sind sie viel weniger empfindlich. Eines der Bakterien ist im Grolen in Reinzucht in Essigbildnern der Versuchsessigfabrik des Institutes für Gärungsgewerbe in Berlin tätig.

Für die Fortzüchtung von Rein. zucht-Essigbakterien und ihre Ubertragung in den Betrieb gibt $\mathrm{F}$. R o t h e $\mathrm{n} \mathrm{b} \mathrm{a} \mathrm{c} \mathrm{h}^{53}$ ) Vorschriften. Die auf Agar oder Gelatine gezüchteten Bakterien haben meist an Säure- und Hantbildungsvermögen gelitten und müssen diese ihre wertvollen Eigenschaften erst durch allmähliche Gewöhnung wiedergewinnen. Die gegebenen Vorschriften beziehen sich sowohl auf Bakterien für das Orleansverfahren wie auch auf solche für die Schnellessigfabrikation. Derselbe Verf. ${ }^{54}$ ) hat vergebliche Versuche angestellt, das $\mathrm{Ox}$ y d a $\mathrm{t}$ i o $\mathrm{n} \mathrm{s}$ vermögen von. Fssigbakterien durch Züchtung bei Gegenwart gewisser Mengen Ferround Manganosalze zu erhöhen.

Die Zymasebildung in der Hefe und damit ihre Gärfähigkelt wird nach H. P r i n $g$ sh e i m ${ }^{55}$ ) sehr stark durch die Konstitution der Stickstoffnahrung beeinflußt, die der Hefe zu Gebote steht. Um gärfähige Hefe zu erhalten, muß man der Hefe Stickstoffkörper mit der Gruppe NH.CH.CO - zur Verfügung stellen, beim Fehlen dieser Gruppe findet in der Zuckerlösung zwar eine schwache Hefenvermehrung statt, es tritt aber keine Gärung ein.

A. H a r d e $\mathbf{n}$ und W. Y o un $\mathbf{g}^{56}$ ) berichten

48) Wochenschr. f. Brauerei 23, 69 (1906).

49) Z. f. Spiritusind. 23, 55 (1906).

50) Diese Z. 18, 610 (1905) (1906)

51) Compt. r. d. Acad. d. sciences 142, 458

52) Essig-Ind. 10, 89 (1906).

53) Essig-Ind. 10, 162 (1906)

54) Essig-Ind. 11, 53 (1907).

$\left.{ }^{55}\right)$ Berl. Berichte 39, 4048 (1906).

56) Proc. Royal Soc. yg, Scrie B 405 (1906) 
ausführlicher über ihre bereits früher erwähnten Versuche ${ }^{57}$ ) betr. E in $\mathrm{flu} \boldsymbol{B}$ von gekochtem und filtriertem Hefesaft auf die Gärwirkung vo $\mathrm{n} \mathrm{H}$ e f e pre Bs a f t. Im Zusammenhang mit diesen Arbeiten steht eine weitere derselben Verf. ${ }^{58}$ ), welche sich mit dem Einfluß der A r s e $n$ a t e a $\mathbf{f}$ die Gärkraft des PreBsaftes beschäftigt. Bekanntlich erhöhen die Phosphate die Gärkraft außerordentlich, und von anderer Seite ${ }^{59}$ ) wurde in dieser Erscheinung die Erklärung für die Einwirkung des gekochten Hefesaftes gesucht. H a r d e n und $\mathrm{Y}$ o u $\mathrm{n}$ g haben nun die Phosphate durch Arsenate ersetzt und dabei eine noch stärkere Reizwirkung beobachten können. Allerdings hört die Vergärung früher auf wie bei fehlendem Zusatz, die entwickelte Kohlensäuremenge ist aber erheblich größer.

Sehr hohe Zuckerkonzentrationen können, wie T. Grom o ${ }^{60}$ ) zeigt, die $\mathrm{Sel} \mathbf{b} \mathrm{st}$ verdaungin abgetöteten Hefezelle $\mathbf{n}$ vollkommen aufheben, ja die Endotryptase der Hefe scheint unter diesen Umständen sogar zur Eiweißsynthese angeregt zu werden. Die Wirkung der I $n$ vert a s e aus einer Anzahl Mikroorganismen wie Hefe, Mucorarten, soll nach E. P a n t a n e 11 i ${ }^{61}$ ) durch Kolloidstoffe, Gelatine, Kieselsäure, arabischcn Gummi herabgesetzt werden, ebenso die Sekretion des Enzyms durch die Organismen. Die Gegenwart von $\mathrm{Emuls}$ in in der $\mathrm{Hefe}^{62}$ ) wird durch L. G u i g $\mathrm{n}$ a $\mathrm{r} \mathrm{d}^{63}$ ) bestätigt. Bäckerhefe und Hefe aus gärendem Holundersaft (Saccharomyces Pastorianus) waren imstande Amygdalin zu spalten. A. B a $\mathrm{c} \mathrm{h}^{\mathbf{6 4}}$ ) berichtet über den Einfluß der Peroxydase auf die alkoholi s ch e Gärung und das Schicksal der Katal a s e bei der zellenfreien Gärung. Das sehr beschränkte Oxydationsvermögen des Systems Peroxydase-Hydroperoxyd, das unter gewöhnlichen Verhältnissen auf Zucker ohne Einfluß ist, erfährt auch durch Gegenwart von Zymase keine Steigerung. Dagegen hemmt aktive Peroxydase die alkoholische Gärung stark. Dic Katalasewirkung der Dauerhefe geht bei der Gärung mit solcher Hefe rasch zurück, störend scheint hier die Anhäufung der Gärungsprodukte $\mathrm{zu}$ wirken.

W. F. B o os ${ }^{65}$ ) hat die bereits bekannte Mykonucleïnsäure aus Hefe nach abgeändertem Verfahren dargestellt und ihre Zusammensetzung als der Formel $\mathrm{C}_{36} \mathrm{H}_{52} \mathrm{~N}_{14} \mathrm{O}_{14} \cdot 2 \mathrm{P}_{2} \mathrm{O}_{5}$ entsprechend ermittelt. Mitteilung über $\mathrm{As} \mathrm{ch}$ en gehalt und $Z$ usammensetzung der Asche einiger H ef eras sen (Rasse II, XII und einer Lufthefe) macht $\mathrm{G}$. H e i $\mathrm{n}$ z e $\mathrm{l}$ m a $n \mathbf{n}^{\mathbf{6}}$ ). Entfettete Essigsäurebazillen enthalten nach E. A lila i r e ${ }^{67}$ ) $6,9 \%$ Stickstoff und

57) Diese Z. 19, 621 (1906).

58) Proc. Chem. Soc. 22, 283 (1906).

59) Diese Z. 19, 621 (1906).

60) Z. physiol. Chem, 48, 87 (1906).

61) Atti R. Accad. dei Linc. Rom. 15, I, 377 (1906).

62) Diese Z. 19, $621(1906)$

63) Bll. des Scienc. Pharmac. 13, 75 (1906).

64) Berl. Berichte 39, 1664,1669 (1906).

65) Ar. exper. Pathol. u. Pharmak. 55, 16 (1906).

66) Jahrb. Ver. Spiritusfabr. 6, 21 (1906).

67) Compt. r. d. Acad. d. seiences 143, $176(1906)$.
5,9\% Asche. Letztere besteht fast zur Hälfte aus Phosphorsäure, unter den Basen spielt Kali die Hauptrolle, ferner Kalk, Eisen, Magnesia, Natron, Kupfer.

Eine Anzahl bemerkenswerter Arbeiten beschäftigt sich mit dem $\mathrm{Ch}$ e $\mathrm{m}$ is $\mathrm{m}$ us des Gärungsvorganges, so z. B. eine Arbeit von H. S c h a d e ${ }^{68}$ ), der gefunden haben wollte, daß Fruchtzucker unter geeigneten Versuchsbedingungen in alkalischer Lösung nach der glatten Formel

$$
\mathrm{C}_{6} \mathrm{H}_{12} \mathrm{O}_{6}=2 \mathrm{C}_{2} \mathrm{H}_{4} \mathrm{O}+2 \mathrm{H}_{2} \mathrm{CO}_{2}
$$

in Acetaldehyd und Ameisensäure zerfalle. Letztere wird in saurer Lösung durch Rhodiummohr in Wasserstoff und Kohlensäure zerlegt, indem man ersteren zur Reduktion des Aldehydes zu Äthylalkohol benutzt, erhielt man als Zuckerspaltungsprodukte Alkohol und Kohlensäure im selben Verhältnis wie bei der alkoholischen Gärung. Tatsächlich verläuft aber, wie eine Nachprüfung der Schadeschen Angaben durch E. Buchner, J. M e i s e $\mathrm{n}$ h e i m e r und H. S c h a d e ${ }^{69}$ ) ergab, die alkalische Spaltung der Fructose ganz wesentlich komplizierter. Acetaldehyd entsteht dabei überhaupt nicht oder höchstens spurenweise, dafür entstehen neben Ameisensäure noch sehr beträchtliche Mengen nicht flüchtiger Säuren, vor allem Glykolsäure und Erythronsäure.

Aus einer anderen Arbeit von F. B u ch n e r und J. Me is e $n h$ ei $m$ e $r^{70}$ ) über die che $m i$ sohen Vorgänge bei der alkoholis ch e n G är u n g mögen folgende Ergebnisse Erwähnung finden: Entgegen früheren Angaben entsteht Bernsteinsäure bei der zellenfreien Gärung nicht, dagegen Glycerin in reichlichen Mengen. Die von anderer Seite geäußerte Annahme, daß das Glycerin seine Entstehung einer lipolytischen Fettspaltung verdanke, kann nicht aufrecht erhalten werden, da sich in der Gärlösúng keine höheren freien Fettsäuren finden. Die Erscheinung, daß bei der zellenfreien Gärung scheinbar mehr Zucker verschwindet als vergoren wird, hat ihre Erklärung darin gefunden, daß ein Teil des Zuckers zu einem nicht reduzierenden Polysaccharid verwandelt wird. Fuselöl entsteht nicht oder höchstens spurenweise. ( $\mathrm{Zu}$ diesem letzten Punkt siehe weiter unten.)

Eine Veröffentlichung von A. S l a to $\mathrm{r}^{71}$ ) befaßt sich mit der chemischen Dynamik deralkoholischen Hefegärung. Der Betrag der gespaltenen Dextrose ist der Hefekonzentration proportional und unabhängig, außer in sehr verdünnten Lösungen, von der Zuckerkonzentration. Der sehr hohe Temperaturkoeffizient der Reaktion schwankt, z. B.

$$
\frac{V_{15}}{V_{5}}=5,6, \quad \frac{V_{40}}{V_{30}}=1,6
$$

Weitere Aufklärungen hat das Berichtsjahr über die F u s e lö l f r a g e gebracht. Die Bildung der Fuselöle aus den Aminos äuren durch die Hefe hängt nach F. E h r li c h 72) ${ }^{73}$ ) mit dem EiweiBaufbau in der Hefe zusammen, das ab-

68) Z. physik. Chem. 5\%, 1 (1906).

69) Berl. Berichte 39, 4217 (1906).

70) Berl. Berichte 39, 3201 (1906).

71) Proc. Chem. Soc. 21, 304 (1906).

72) Biochem. Zeitschr. 2, 52 (1906).

73) Berl. Berichte 39, 4072 (1906). 
gespaltene Ammoniak wird von der Hefe zu diesem Zweck aufgenommen. DemgemäB setzt die Zugabe von Ammoniakstickstoff zu den gärenden Flüssigkeiten die Fuselölbildung herab. Die Umwandlung der Aminosäuren geschieht nur durch lebende Hefe, nicht durch Preßsaft oder Dauerhefe. Die letztere Beobachtung hat auch $\mathrm{H}$. Pring s h e i m ${ }^{\mathbf{7 4}}$ ) gemacht, der vergeblich versuchte, bei Gärung mittels Acetondauerhefe durch Zugabe von Leucin Amylalkohol zu erhalten. Die Einwirkung der Hefe auf Aminosäuren kann nach F. E h r li $\mathrm{ch}^{75}$ ) zur Trennung racemischer Aminosäuren und Gewinnung von optisch aktiven Aminosäuren benutzt werden. Unter geeigneten Versuchsbedingungen wird aus dem Racemat nur eine optischaktive Modifikation in Alkohol umgewandelt, während die entgegengesetzte nach Beendigung der Gärung leicht in reinem Zustand aus der Gärflüssigkeit isoliert werden kann.

Die $S$ chwefelwasserstoff bild ung d u r ch $\mathrm{H}$ ef en ${ }^{76}$ ) hängt nach $\mathrm{H}$. Will und $\mathrm{H}$. W anderscheck ${ }^{77}$ ) ihrer Stärke nach sehr von Hefenart und -rasse (außer ober- und untergärigen Brauereihefen gelangte eine italienische Weinhefe und verschiedene wilde Hefen zur Untersuchung) und von der Zusammensetzung der Würze ab. Die eigentliche Schwefelwasserstoffquelle ist noch nicht sicher, Peptonzusatz vermindert die Bildung. In mineralischen Nährsalzlösungen mit Zucker und Asparagin findet viel stärkere Entwicklung statt wie in gehopften Würzen, Schwefelzusatz erhöht die Bildung. In einer ausführlichen Veröffentlichung fassen $\mathrm{E}$. $\mathrm{B}$ u $\mathrm{ch} \mathrm{ner}$ und $R$. Ga $a n$ t $^{78}$ ) die bislang erzielten Ergebnisse über die enzymatische Essiggärung zusammen. Von neueren Beobachtungen, über die in der Veröffentlichung berichtet wird, ist zu erwähnen vor allem der Einflu $ß$ der Kulturbedingungen der Essigbakterien auf das Oxydationsvermögen der daraus gewonnenen Dauerbakterien. Auch im günstigsten Fall ist dasselbe recht gering, indem auf $100 \mathrm{~g}$ Dauerbakterien höchstens $4 \mathrm{~g}$ Essigsäure erhalten wurden. Die Versuche zur Gewinnung von wirksamem Preßsaft aus den Bakterien scheiterten.

Auch einige rein $\mathrm{tech} \mathrm{n}$ is che A r beiten, die unter die Uberschrift Gärungsorganismen und Gärungsvorgang gehören, mögen hier Erwähnung finden. Die nach den üblichen Methoden ziemlich zeitraubende Bestimmung des Endverg är ung s g r a d e s in Würzen, Bier läßt sich nach F. S ch o en fel $\mathrm{d}^{79}$ ) innerhalb 24 Stunden durchführen, wenn man die zu untersuchende Flüssigkeit mit 10\% Hefe anstellt und die Gärung im Thermostaten bei $25^{\circ}$ verlaufen läßt. Zur $\mathrm{H}$ e r stellung von WeiBbier leitet O. Franke ${ }^{80}$ ) in der Maische oder Würze zunächst eine Säuerung durch Milchsäurebakterien ein; nach Erreichung des gew ünschtcn Säuregrades werden die Bakterien durch Pasteurisieren abgetötet und die sterile Würze mit Reinhefe zur alkoholischen Gärung angestellt.

74) Berl. Berichte 39, 3713 (1906).

$75)$ Z. Ver. d. Rübenzucker-Ind. 56, 840 (1906).

$\left.{ }^{76}\right)$ Diese Z. 19, 622 (1906).

77) Z. ges. Brauwesen 29, 73 (1906).

78) Liebigs Ann. 349, 140 (1906).

79) Wochenschr. f. Brauerei 23, 489 (1906).

${ }^{80)}$ Wochenschr. f. Brauerei 23, 384 (1906).
Die großen Unterschiede in der Triebkraft von Bierhefe und Preßhefe bei höh erer Temperatur, über die früher berichtet ${ }^{81}$ ) wurde, werden nach $\mathrm{H}$. Lange ${ }^{82}$ ) noch viel augenfälliger, wenn der Gärflüssigkeit Reizstoffe wie Ammoniumphosphat, Kaliumphosphat, Magnesiumsulfat, Asparagin in passenden Mengen zugesetzt werden. Zur Erzeugung vongärkräfti ger Pre $B$ he fe in Reinzucht benutzt K. Kr u is ${ }^{83}$ ) ein sterilisiertes Gemisch von stärkefreiem Kartoffelsaft mit Rübensaft. Vor dem Sterilisieren wird das Gemisch, dem noch geeignete Nährstoffe zugesetzt werden können, mit Schwefelsäure angesäuert. G. K ö c k ${ }^{84}$ ) beschreibt einen neuen HefetriebkraftBestimmungsapparat, bei dem die Triebkraft an der Drucksteigerung im Gärgefäß gemessen wird. Für Verwertung der Brauereihefe werden verschiedene Vorschläge gemacht: Die Wissenschaftliche Station für Brauerei in München ${ }^{85}$ ) will sie in ein $\mathrm{Trock}$ en futtermittel verwandeln, indem sie sie kurze Zeit mit etwas Kochsalz stehen läBt, dann auf $75^{\circ}$ erhitzt und mit anderen als Futtermittel geeigneten Stoffen mischt. Der Mi schung wird dann zur Auflockerung und Ermöglichung eines raschen Trocknens kurz zerschnittenes Stroh zugesetzt. Ph. Schidrowitz und F. $\mathrm{K}$ a $\mathrm{y} \mathrm{e}^{86}$ ) machen den Vorschlag, die Hefe durch trockene Destillation zu verwerten. Die Produkte sind ammoniakreiches Wasser, ein dem Knochenteer ähnlicher Teer und ein an Kali, Phosphorsäure und Stickstoff reicher Koks.

\section{Gärungserzeugnisse: Bier, Alkohol, Essig.}

Die zahlreichen Veröffentlichungen ${ }^{87}$ ) über den EinfluB der Metalle a u $B$ ier vermehren L. $N$ a th a $n$ und $A . S$ e h $m$ i d $t^{88}$ ) um eine weitere. Die Reihenfolge der untersuchten Metalle resp. Metallpaare ist, nach der ihrer Schädlichkeit geordnet, mit dem schädlichsten beginnend, folgende : Zinn, Zinn + Aluminium, Zinn + Kupfer, Eisen, Zinn + Zink, Nickelstahl, Zink, Eisen + Kupfer, Nickel, nickelplatierter Flußstahl, Weißblech,Zinn + Blei, Zink + Kupfer, Nickel + Kupfer.

Für das $N$ a ch d unkeln heller Biere ist nach C. B l e i s c h und K. R u n e k ${ }^{89}$ ) die Zusammensetzung des Wassers von besonderer Bedeutung, ungünstig wirkt vor allem Calciumearbonat. Unter den Bierbestandteilen spielt besonders der Hopfen eine Rolle, je blasser dieser ist, desto geringer ist die Zufärbung. Einflüsse, die sich bei der Bierbereitung ungünstig bemerkbar machen, sind große Pfannen mit tiefen Würzeschichten und Lüftung.

Eine etwas modifizierte Bi eran a l ys e auf Extrakt und Alkohol schlägt H. S t a d l in g e r ${ }^{90}$ )

81) Diese Z. 19, 619 (1906).

82) Brennereizeitung 1906, 4028.

83) D. R. P. 173231.

84) Z. österr. landw. Vers.-Wes. 9, 801 (1906)

$85)$ D. R. P. 174864.

86) J. Inst. Brew. 1906, 450.

87) Diese Z. 18, 613 (1905); 19, 622 (1906).

88) Z. Bakt. u. Paras. 16, II, 482 (1906).

89) Z. ges. Brauwesen 29, 277 (1906).

${ }^{90)}$ Z. ges. Brauwesen 29, 624 (1906). 
vor. Er destiliert $75 \mathrm{ccm}$ Bier, fängt $50 \mathrm{ccm}$ Alkoholdestillat auf und verdünnt den Rückstand ebenfalls auf $50 \mathrm{ccm}$. Die erhaltenen spezifischen Gewichte werden mit Hilfe von Tabellen in Extraktund Alkoholgehalt in $100 \mathrm{ccm}$ Bier umgewertet, mit Hilfe des spezifischen Gewichtes des Bieres lassen sich weiter die Gewichtsprozente berechnen. Worin die Vorteile dieser Methode gegenüber der bisher üblichen bestehen sollen, ist nicht recht klar. $\mathrm{P} . \mathrm{M} n \mathrm{~mm} \mathrm{e}^{91}$ ) teilt die An a 1 y se von D a n $\mathrm{z}$ i g e r J o p e n b i e r mit, dasselbe enthält $3,52 \%$ Alkohol und $45,4 \%$ wirklichen Extrakt. Der wirkliche Vergärungsgrad beträgt nur 9,81\%. Bei der Kohlensäurebestimm ung im Bier wollen $O$. R e i $\mathrm{nk}$ e und $\mathrm{A}$. W i e b o $1 \mathrm{~d}^{92}$ ) die ausgetriebene Kohlensäure volumetrisch bestimmen.

Bei einer Trennung der Stickst of $\mathbf{f} s \mathrm{u}$ stanzen im Bier hat $O$. Miskovsky ${ }^{93}$ ) in einem Pilsener Bier mit 39,96 $\mathrm{g}$ Trockensubstanz in 11 folgende Ergebnisse erhalten : 1,175 g Eiweiß nach $R \ddot{\mathrm{u}} \mathrm{m} \mathrm{p} \mathrm{l}$ e r , 0,700 g nach S t u t z e r , 0,078g Cholin, 0,033 g Betain, ca. 0,005 g Arginin, 0,002 g Histidin.

Ein neues Verfahren, A $1 \mathrm{k}$ o ho 1 o h ne G ä r ung zu gewinnen, wird von Donas, Desm o n t s und Deglatign $y^{94}$ ) in Vorschlag gebracht. Acetylen soll von Quecksilbersalzlösungen absorbiert werden, das entstehende Acetylenquecksilber zersetzt sich beim Kochen unter Entwicklung von Acetaldehyd, welch letzterer mittels Alkaliamalgam zu Athylalkohol reduziert werden soll. Es muß zugegeben werden, daß sich vielleicht noch umständlichere Verfahren zur Alkoholgewinnung werden ausfindig machen lassen, über den Preis des synthetischen Alkohols verraten die Erfinder nichts.

Die unangenehm empyreumatisch riechenden Verunreinigungen in Rohspiritus a us L üftu $\mathrm{n} g \mathrm{~s} w$ ür z en lassen sich nach $\mathrm{Ch} \mathbf{r}$. A. Petersen-Schepelern und C. W. $\mathrm{S}$ c hw a n e $\mathrm{n} \mathbf{1} \mathbf{1}$ ü g e l ${ }^{95}$ ) durch gleichzeitige Behandlung dieses Spiritus mit Holzkohle und Wasserstoffsuperoxyd entfernen.

Ein Verfahren nach $\mathrm{A}$. K $0 \mathrm{ch}^{\mathrm{96}}$ ) bezweckt die Gewinnung fuselölarmer oder fusel. freier vergorener Flüssigkeiten. Es besteht darin, daß die gärende Flüssigkeit unter Einleiten von elementarem Sauerstoff mit Influenzelektrizität behandelt wird. Die dabei sich geltend machende Ozonbildung soll die Gärtätigkeit nicht schädigen, ferner sollen aber unter ihrem Einfluß Stoffe entstehen, die sich sonst nur bei langem Lagern der Flüssigkeiten bilden.

Zur Herstellung voll $\mathrm{k}$ o m men w a s e r freien Alk ohols ${ }^{97}$ ) behandelt man hochkonzentrierten Alkohol mit Calciumspänen. Die aus verunreinigendem Calciumnitrid stammenden Spuren Ammoniak werden dem Alkohol durch Behandeln mit Säure oder sauer wirkenden Salzen

91) Wochenschr. f. Brauerei 23, 13 (1906).

92) Chem. Zeitung 30, 1261 (1906).

93) Z. ges. Brauwesen 29, 309 (1906).

94) Z. f. Spiritusind. 29, 303 (1906):

95) D. R. P. 170121.

96) D. R. P. 170167 .

97) D. R. P. 175780 ; siehe auch diese Z. 19, $623(1906)$. entzogen, z. 13. indem man die Alkoholdämpfe kei der Destillation über gebrannten Alaun leitet.

Nach F. Karasef $f{ }^{98}$ ) erhält man ein zur Denaturierung von Spiritus geeig$\mathrm{n}$ etes $\mathrm{K}$ eto $\mathrm{n} \ddot{\mathrm{l}} \mathrm{l}$, wenn man Brennereimaischen bei Gegenwart ven Calciumcarbonat einer sauren Gärung überläßt und die erhaltenen Kalksalze der trockenen Destillation unterwirft. Selbst wenn sich das erhaltene Mittel nach jeder Richtung hin als Denaturierungsmittel eignen sollte, dïrfte die Eerstellung des Ketonöls viel zu teuer sein, um mit den üblichen Denaturierungsmitteln konkurrieren zu können.

E. Schmid t ${ }^{99}$ ) will Gärungsessig von Essigessenzessig dadurch unterscheiden, daß er den betreffenden Essig mit Natronlauge alkalisch macht, mit Amy]alkohol ausschüttelt und nach dem Verdampfen des letzteren den Rückstand mit Wasser aufnimmt, mit Schwefelsäure ansäuert und Jodjodkalium zugibt. Trübung oder Niederschlag zeigt Gärungsessig an, die Reaktion scheint von Bakterienstoffwechselprodukten verursacht $\mathrm{zu}$ werden. Im übrigen hat die Methode verzweifelt viel Âhnlichkeit mit einer von $\mathrm{P}$ a r o w ${ }^{100}$ ) angegebenen.

Chemische Daten lassen nur eine unsichere $\mathbf{B}$ e urteilung von Weinessig zu, wie $A$. Jons $c h$ e $\mathbf{r}^{101}$ ) ausführt, speziell ist es nach $F$. R ot h e n b a c h 102) verfehlt, den Glyceringehalt der Weinessige zur Grundlage der Beurteilung zu machen; die Versuche zeigten, daß ein Teil des Glycerins bei der Gärung verschwindet

\section{Über die \\ quantitative Bestimmung von Amido= und Hydroxylverbindungen der Benzol- und Naphthalinreihe.}

\author{
Von HaNs Th. Bucherer. \\ (Eingeg. A. 2.3. 1907.)
}

Unter den Zwischenprodukten für die Darstellung organischer Farbstoffe nehmen die $\mathrm{Ab}$ kömmlinge der Benzol- und Naphthalinreihe eine sehr wichtige Stellung ein. Dies gilt insbesondere auch von solchen Verbindungen der aromatischen Reihe, die infolge des Vorhandenseins auxochromer Gruppen durch besondere Reaktionsfähigkeit ausgezeichnet sind (R.OH, R. $\mathrm{NH}_{2}, \mathrm{R} . \mathrm{NH}$ Alkyl, R.NH Aryl usw.). Die Darstellungsweise einerseits und die Eigenschaften dieser Körper andererseits bringen es mit sich, daB die Technik in sehr vielen Fällen darauf verzichten $\mathrm{mu} \beta$ und auch darauf verzichten kann, sie im Zustand absoluter Reinheit, d. h. in Form eines etwa 100\%igen Produktes weiter zu verarbeiten. In manchen Fällen sieht sie sich genötigt, mit Lösungen zu operieren, wie sie sich bei der Darstellung unmittelbar ergeben; in anderen Fällen mit wasserhaltigen Pasten oder Preßkuchen, und wiederum in anderen Fällen erweist

98) D. R. P. 175078 .

99) Z. Unters. Nahr.- u. Genu Bm. 11, 386 (1906).

100) Essig-Ind. 6, 49 (1902).

101) Z. öff. Chem. 11, 467 (1906).

102) Essig-Ind. 11, 54 (1907). 